\title{
The ADAMTS13-VWF axis is dysregulated in chronic thromboembolic pulmonary hypertension
}

\author{
Michael Newnham ${ }^{1,2}$, Kieron South ${ }^{3}$, Marta Bleda', William R. Auger ${ }^{4}$, \\ Joan A. Barberà ${ }^{5}$, Harm Bogaard ${ }^{6}$, Katherine Bunclark², John E. Cannon², \\ Marion Delcroix (10 ${ }^{7}$, Charaka Hadinnapola ${ }^{1}$, Luke S. Howard ${ }^{8}$, David Jenkins ${ }^{2}$, \\ Eckhard Mayer ${ }^{9}$, Choo $\mathrm{Ng}^{2}$, Christopher J. Rhodes ${ }^{10}$, Nicholas Screaton ${ }^{2}$, \\ Karen Sheares ${ }^{2}$, Michael A. Simpson ${ }^{11}$, Mark Southwood ${ }^{2}$, Li Su ${ }^{12}$, \\ Dolores Taboada ${ }^{2}$, Matthew Traylor ${ }^{13}$, Richard C. Trembath ${ }^{11}$, Sofia S. Villar ${ }^{12}$, \\ Martin R. Wilkins ${ }^{10}$, John Wharton ${ }^{10}$, Stefan Gräf (1) ${ }^{1,14,15}$, Joanna Pepke-Zaba², \\ Michael Laffan ${ }^{3}$, David A. Lane ${ }^{3}$ Nicholas W. Morrell ${ }^{1}$ and Mark Toshner ${ }^{1,2}$
}

@ERSpublications

The ADAMTS-13-VWF axis is dysregulated in chronic thromboembolism with and without pulmonary hypertension and is implicated in the pathogenesis http://ow.ly/J9SC30nh5T0

Cite this article as: Newnham M, South K, Bleda M, et al. The ADAMTS13-VWF axis is dysregulated in chronic thromboembolic pulmonary hypertension. Eur Respir J 2019; 53: 1801805 [https://doi.org/10.1183/ 13993003.01805-2018].

ABSTRACT Chronic thromboembolic pulmonary hypertension (CTEPH) is an important consequence of pulmonary embolism that is associated with abnormalities in haemostasis. We investigated the ADAMTS13-von Willebrand factor (VWF) axis in CTEPH, including its relationship with disease severity, inflammation, $A B O$ groups and ADAMTS13 genetic variants.

ADAMTS13 and VWF plasma antigen levels were measured in patients with CTEPH ( $\mathrm{n}=208)$, chronic thromboembolic disease without pulmonary hypertension (CTED) $(\mathrm{n}=35)$, resolved pulmonary embolism $(\mathrm{n}=28)$, idiopathic pulmonary arterial hypertension $(\mathrm{n}=30)$ and healthy controls $(\mathrm{n}=68)$. CTEPH genetic $A B O$ associations and protein quantitative trait loci were investigated. ADAMTS13-VWF axis abnormalities were assessed in CTEPH and healthy control subsets by measuring ADAMTS13 activity, Ddimers and VWF multimeric size.

Patients with CTEPH had decreased ADAMTS13 (adjusted $\beta-23.4 \%, 95 \%$ CI $-30.9--15.1 \%, \mathrm{p}<0.001$ ) and increased VWF levels $(\beta+75.5 \%, 95 \%$ CI $44.8-113 \%, \mathrm{p}<0.001)$ compared to healthy controls. ADAMTS13 levels remained low after reversal of pulmonary hypertension by pulmonary endarterectomy surgery and were equally reduced in CTED. We identified a genetic variant near the ADAMTS13 gene associated with ADAMTS13 protein that accounted for $\sim 8 \%$ of the variation in levels.

The ADAMTS13-VWF axis is dysregulated in CTEPH. This is unrelated to pulmonary hypertension, disease severity or markers of systemic inflammation and implicates the ADAMTS13-VWF axis in CTEPH pathobiology. 


\section{Introduction}

Chronic thromboembolic pulmonary hypertension $(\mathrm{CTEPH})$ results from failure of thrombus resolution in the pulmonary arteries following acute pulmonary embolism (PE) in 3\% of cases [1]. Organisation and fibrosis of thrombotic material leads to the obstruction of proximal pulmonary arteries and the subsequent development of a secondary small vessel vasculopathy, both of which contribute to pulmonary hypertension and subsequent right heart failure $[2,3]$.

Abnormalities in haemostasis are implicated in CTEPH pathobiology $[4,5]$. This includes elevated von Willebrand factor (VWF), a multimeric plasma glycoprotein that is synthesised by vascular endothelial cells and megakaryocytes $[6,7]$. VWF plays an important role in platelet recruitment by mediating the adhesion of platelets to the endothelium and is also a carrier protein for the pro-coagulant blood clotting Factor VIII [7]. VWF activity is normally regulated by ADAMTS13 (a disintegrin and metalloproteinase with a thrombospondin type 1 motif, member 13), a plasma protein that specifically cleaves the more active high molecular weight VWF multimers [8]. ADAMTS13 is predominately produced by hepatic stellate cells, in addition to vascular endothelial cells and megakaryocytes [9]. The critical role of ADAMTS13 levels in haemostasis is exemplified by thrombotic thrombocytopenic purpura (TTP), characterised by micro-angiopathic thrombosis, in which plasma levels of ADAMTS13 are severely reduced by autoantibodies or rare ADAMTS13 mutations [10].

Plasma VWF levels are increased in a range of thrombotic conditions, including coronary artery disease (CAD), ischaemic stroke and venous thromboembolism [11, 12]. Conversely, plasma ADAMTS13 levels are modestly reduced in CAD and ischaemic stroke $[11,13]$. There are discordant findings in patients with acute PE, with ADAMTS13 levels reported to be increased, no different and decreased [14-16]. VWF and Factor VIII are known to be elevated in CTEPH and do not change following pulmonary endarterectomy (PEA), suggesting a role in pathogenesis [6]. Whilst VWF cleaving protease has been indirectly assessed in CTEPH, the direct role of ADAMTS13 has not been investigated to date [6].

A large proportion of the variation in VWF levels is genetically determined, with $30 \%$ due to $A B O$ groups [17]. The ADAMTS13 gene is situated $\sim 200$ kilobases $(\mathrm{kb})$ downstream of $A B O$ and is genetically regulated, with $20 \%$ of its variance attributable to common variants at the ADAMTS13 locus [18]. ADAMTS13 is not known to vary with $A B O$ groups in healthy cohorts [19]. Similar to in other thrombotic diseases, the non-O blood groups are over-represented in CTEPH, suggesting a mechanism by which VWF levels are increased [20]. We aimed to investigate the ADAMTS13-VWF axis in CTEPH patients, including its relationship with $A B O$ groups and ADAMTS13 genetic variants.

\section{Methods}

\section{Study samples and participants}

The study was approved by the regional ethics committee (REC no. 08/H0304/56 and 08/H0802/32) and all study participants provided written informed consent from their respective institutions.

Consecutive CTEPH patients from the national PEA centre (Royal Papworth Hospital, Cambridge, UK) with available plasma samples (August 2013-December 2016) (supplementary figure S1) and genotype data were included in the study $(n=208)$. Healthy volunteers $(n=68)$ were used as a control group (Royal Papworth Hospital and Hammersmith Hospital, London, UK). Additional patient groups were recruited as disease comparators, including patients with chronic thromboembolic disease (CTED) ( $n=35)$, idiopathic pulmonary arterial hypertension $($ IPAH $)(n=30)$ and PE $(n=28)$. CTED was characterised by persistent pulmonary arterial thromboembolic occlusions without pulmonary hypertension (mean pulmonary arterial pressure $<25 \mathrm{mmHg}$ ) in symptomatic patients, and other diagnoses were made using international criteria $[21,22]$.

Affiliations: ${ }^{1}$ Dept of Medicine, University of Cambridge, Addenbrooke's Hospital, Cambridge, UK. ${ }^{2}$ Royal Papworth Hospital, Cambridge, UK. ${ }^{3}$ Centre for Haematology, Imperial College London, London, UK. ${ }^{4}$ University of California, San Diego, CA, USA. ${ }^{5}$ Hospital Clínic - IDIBAPS-CIBER Enfermedades Respiratorias, University of Barcelona, Barcelona, Spain. ${ }^{6}$ VU University Medical Centre, Amsterdam, The Netherlands. ${ }^{7} \mathrm{KU}$ Leuven - University of Leuven, Leuven, Belgium. ${ }^{8}$ Hammersmith Hospital, Imperial College Healthcare NHS Trust, London, UK. ${ }^{9}$ Kerckhoff Heart and Lung Centre, Bad Nauheim, Germany. ${ }^{10}$ Centre for Pharmacology and Therapeutics, Dept of Medicine, Hammersmith Campus, Imperial College London, London, UK. ${ }^{11}$ Dept of Medical and Molecular Genetics, King's College London School of Basic and Medical Biosciences, London, UK. ${ }^{12}$ MRC Biostatistics Unit, School of Clinical Medicine, University of Cambridge, Cambridge, UK. ${ }^{13}$ Dept of Clinical Neurosciences, University of Cambridge, Cambridge, UK. ${ }^{14}$ Dept of Haematology, National Health Service Blood and Transplant Centre, University of Cambridge, Cambridge, UK. ${ }^{15}$ National Institute of Health Research BioResource for Translational Research, Cambridge Biomedical Campus, Cambridge, UK.

Correspondence: Mark Toshner, Dept of Medicine, University of Cambridge, School of Clinical Medicine, Box 157, Addenbrooke's Hospital, Hills Road, Cambridge CB2 OQQ, UK. E-mail: mrt34Amedschl.cam.ac.uk 


\section{ADAMTS13 and VWF plasma concentrations}

Plasma samples were used to measure ADAMTS13 and VWF antigen (Ag) levels by ELISA. Samples for the CTEPH, CTED and IPAH groups were obtained closest to the time of diagnosis, and pre-operatively for the CTEPH and CTED patients undergoing PEA. Additionally, ADAMTS13 and VWF levels were measured in 22 paired post-PEA samples taken within 1 year of surgery to assess the effect of PEA. The PE group was sampled from a specialist PE follow-up service (Hammersmith Hospital, London) at a median (interquartile range (IQR)) of 220 (218) days following an acute PE.

ADAMTS13 and VWF plasma antigen levels were quantified using polyclonal rabbit anti-ADAMTS13 and anti-VWF antibodies as previously described (supplementary material) [19, 23].

ADAMTS13 activity, D-dimer, anti-ADAMTS13 autoantibodies and VWF multimeric size

Additional experiments were performed on a subset of the CTEPH $(n=21-23)$ and healthy control $(n=14)$ groups to identify potential mechanisms for any dysregulation of the ADAMTS13-VWF axis. Plasma samples were used to measure ADAMTS13 activity (fluorescence resonance energy transfer assay), D-dimer concentrations (ELISA) and anti-ADAMTS13 autoantibodies (CTEPH, n=23), with further details in the supplementary material. An estimate of VWF multimeric size was made by measuring VWF collagen binding (VWF collagen binding assay (CBA)) and comparing this with VWF antigen levels (CTEPH, $\mathrm{n}=21)$.

\section{Clinical phenotype data}

Phenotype data for the CTEPH, CTED and IPAH groups were recorded closest to the time of diagnosis and pre-operatively for the CTEPH and CTED patients undergoing PEA. This included demographics, haemodynamics, World Health Organization functional class, 6-minute walk distance (6MWD), clinical blood tests, smoking history and anticoagulation therapy usage.

\section{Genotype data}

Imputed genotype dosages were available from an ongoing international genome-wide association study (GWAS) in CTEPH that will be published separately on recruitment of a validation cohort. All individuals were genotyped on commercially available Illumina assays (Illumina, Cambridge, UK) and imputed to the Haplotype Reference Consortium build 1.1 [24]. Additional details and quality controls steps are described in the supplementary material.

Genotypes were available for 207 patients (185 CTEPH, 22 CTED) after GWAS quality control exclusions. These patients were included in the genetic $A B O$ group and protein quantitative trait loci (pQTL) analyses. Matched genotypes and ADAMTS13/VWF antigen levels were not available for the healthy control, IPAH or PE groups.

\section{Genetic $A B O$ groups}

The $A B O$ groups $\mathrm{A} 1, \mathrm{~A} 2, \mathrm{~B}$ and $\mathrm{O}$ were reconstructed using haplotypes from phased data and a described list of tagging $A B O$ single nucleotide polymorphisms (SNPs) (supplementary material). This resulted in 10 groups (A1A1, A1A2, A1B, A1O, A2A2, A2B, A2O, BB, BO, OO), from which blood groups $\mathrm{A}, \mathrm{B}, \mathrm{AB}$ and $\mathrm{O}$ were inferred.

\section{Protein quantitative trait loci}

Associations between genetic variants in the ADAMTS13 gene $\pm 40 \mathrm{~kb}$ ( $\mathrm{n}=396$ variants) and ADAMTS13 protein levels were evaluated using multivariable linear regression. The model was adjusted for age, sex and ADAMTS13 plasma antigen experimental batch. Additional models were adjusted for VWF antigen levels and the first five ancestry informative principal components used in the GWAS analysis. The ADAMTS13 $\pm 40 \mathrm{~kb}$ region included the ADAMTS13 cis-pQTLs that have previously been described $[18,25,26]$.

\section{Statistical analysis}

Group differences in ADAMTS13 and VWF antigen levels were assessed using multivariable linear regression adjusted for age, sex, experimental batch (batch1 versus batch2) and self-reported ethnicity (Caucasian versus non-Caucasian). The $\beta$ coefficients and confidence intervals are presented as percentage change.

Data are presented as median (IQR). Spearman's rank correlation coefficients were used to describe associations between ADAMTS13 or VWF protein levels and clinical phenotypes associated with disease severity and blood markers of inflammation. 


\section{Results}

Baseline group characteristics are summarised in table 1 and supplementary table S1. Age and sex differed across the groups $(\mathrm{p}<0.001$ and $\mathrm{p}=0.014)$, with CTEPH patients being older (64 (19) years) than healthy controls (49 (24) years). Ethnicity also differed $(\mathrm{p}<0.001)$ with more non-Caucasians in the PE group. In the CTEPH group, 176 patients $(87 \%)$ had a proximal distribution of pulmonary arterial obstruction deemed to be surgically accessible and 150 (72\%) underwent PEA.

\section{ADAMTS13 plasma concentrations}

ADAMTS13 antigen levels were decreased in CTEPH patients $\left(0.889(0.397) \mu \mathrm{g} \cdot \mathrm{mL}^{-1}, \mathrm{p}<0.001\right)$ compared to healthy controls $\left(1.15(0.300) \mu \mathrm{g} \cdot \mathrm{mL}^{-1}\right)$ (figure 1a). ADAMTS13 was also reduced in CTED (0.831 (0.224) $\left.\mu \mathrm{g} \cdot \mathrm{mL}^{-1}, \mathrm{p}<0.001\right)$ but levels were similar to those in CTEPH $(\mathrm{p}=0.205)$ (supplementary table S2). There was no difference in ADAMTS13 levels between IPAH $\left(1.12(0.413) \mu \mathrm{g} \cdot \mathrm{mL}^{-1}, \mathrm{p}=0.373\right)$ and healthy controls, though the PE group did exhibit slightly lower levels $\left(0.969(0.704) \mu \mathrm{g} \cdot \mathrm{mL}^{-1}\right.$, $\mathrm{p}=0.049$ ).

Multivariable linear regression confirmed that ADAMTS13 was lowest in the CTEPH ( $\beta$ 23.4\%, 95\% CI $-30.9--15.1 \%, \mathrm{p}<0.001)$ and CTED $(\beta-25.9 \%, 95 \% \mathrm{CI}-35.1--15.4 \%, \mathrm{p}<0.001)$ groups (supplementary table S3). These observations should be interpreted with the additional models utilising interaction terms presented in the supplementary materials. Increasing age was also associated with lower ADAMTS13 ( $\beta-5.06 \%, 95 \%$ CI $-2.99--7.08 \%$ per 10 years, $\mathrm{p}<0.001)$. ADAMTS13 antigen levels were not significantly associated with the PE group $(\beta-12.0 \%, 95 \%$ CI $-24.0-1.97 \%, p=0.089)$ nor IPAH, sex or ethnicity.

\begin{tabular}{|c|c|c|c|c|c|}
\hline & Healthy control & CTEPH & CTED & IPAH & PE \\
\hline Subjects $n$ & 68 & 208 & 35 & 30 & 28 \\
\hline Age years & $49(24)$ & 64 (19) & $58(27)$ & $64(27)$ & $52(26)$ \\
\hline Female sex & 32 (47\%) & $90(43 \%)$ & $9(26 \%)$ & $21(70 \%)$ & $15(54 \%)$ \\
\hline Caucasian ethnicity & 53 (78\%) & $180(95 \%)$ & $28(88 \%)$ & $26(90 \%)$ & $13(54 \%)$ \\
\hline \multicolumn{6}{|l|}{ WHO functional class } \\
\hline 1 & & $4(2 \%)$ & $6(18 \%)$ & $5(17 \%)$ & \\
\hline 2 & & $42(21 \%)$ & $17(50 \%)$ & $4(13 \%)$ & \\
\hline 3 & & $151(74 \%)$ & $11(32 \%)$ & $21(70 \%)$ & \\
\hline 4 & & $7(3 \%)$ & $0(0 \%)$ & $0(0 \%)$ & \\
\hline 6MWD m & & $318(176)$ & $366(180)$ & 342 (244) & \\
\hline \multicolumn{6}{|l|}{ Pulmonary haemodynamics } \\
\hline $\mathrm{mPAP} \mathrm{mmHg}$ & & 42 (18) & $21(4)$ & 42 (17) & \\
\hline Cardiac index $\mathrm{L} \cdot \mathrm{min}^{-1} \cdot \mathrm{m}^{-2}$ & & $2(0.6)$ & $2.4(0.6)$ & $1.7(0.8)$ & \\
\hline PVR dynes $\cdot \mathrm{s} \cdot \mathrm{cm}^{-5}$ & & $639(476)$ & $151(71)$ & 808 (642) & \\
\hline \multicolumn{6}{|l|}{ Clinical blood tests } \\
\hline Haemoglobin $\mathrm{g} \cdot \mathrm{L}^{-1}$ & & 140 (27) & $138(16)$ & $142(22)$ & \\
\hline Platelet count $\times 10^{9}$ & & $246(82)$ & $200(56)$ & $222(77)$ & \\
\hline WCC $\times 10^{9}$ & & $7(3)$ & $6.6(2.1)$ & $6.9(2.4)$ & \\
\hline Lymphocyte \% & & $25(10)$ & 28 (13) & $18(13)$ & \\
\hline Neutrophil \% & & $64(14)$ & $59(14)$ & $72(14)$ & \\
\hline CRP $\mathrm{mg} \cdot \mathrm{L}^{-1}$ & & $5(10)$ & $3(3)$ & $3(4)$ & \\
\hline NT-proBNP pg.mL $\mathrm{mL}^{-1}$ & & $592(1576)$ & 113 (194) & 334 (695) & \\
\hline \multicolumn{6}{|l|}{ Smoking status } \\
\hline Never & & $91(47 \%)$ & $16(50 \%)$ & $15(52 \%)$ & \\
\hline Ex-smoker & & $87(45 \%)$ & $13(41 \%)$ & $11(38 \%)$ & \\
\hline Current smoker & & $15(8 \%)$ & 3 (9\%) & $3(10 \%)$ & \\
\hline Anticoagulation medication & & $137(94 \%)$ & 15 (94\%) & $30(100 \%)$ & \\
\hline
\end{tabular}

Data are presented as median (interquartile range) or $\mathrm{n}(\%)$. Percentages were calculated using the number of patients for whom data were available as the denominator. CTEPH: chronic thromboembolic pulmonary hypertension; CTED: chronic thromboembolic disease; IPAH: idiopathic pulmonary arterial hypertension; PE: pulmonary embolism; WHO: World Health Organization; 6MWD: 6-minute walk distance; mPAP: mean pulmonary arterial pressure; PVR: pulmonary vascular resistance; WCC: white cell count; CRP: C-reactive protein; NT-proBNP: N-terminal pro-brain natriuretic peptide. 

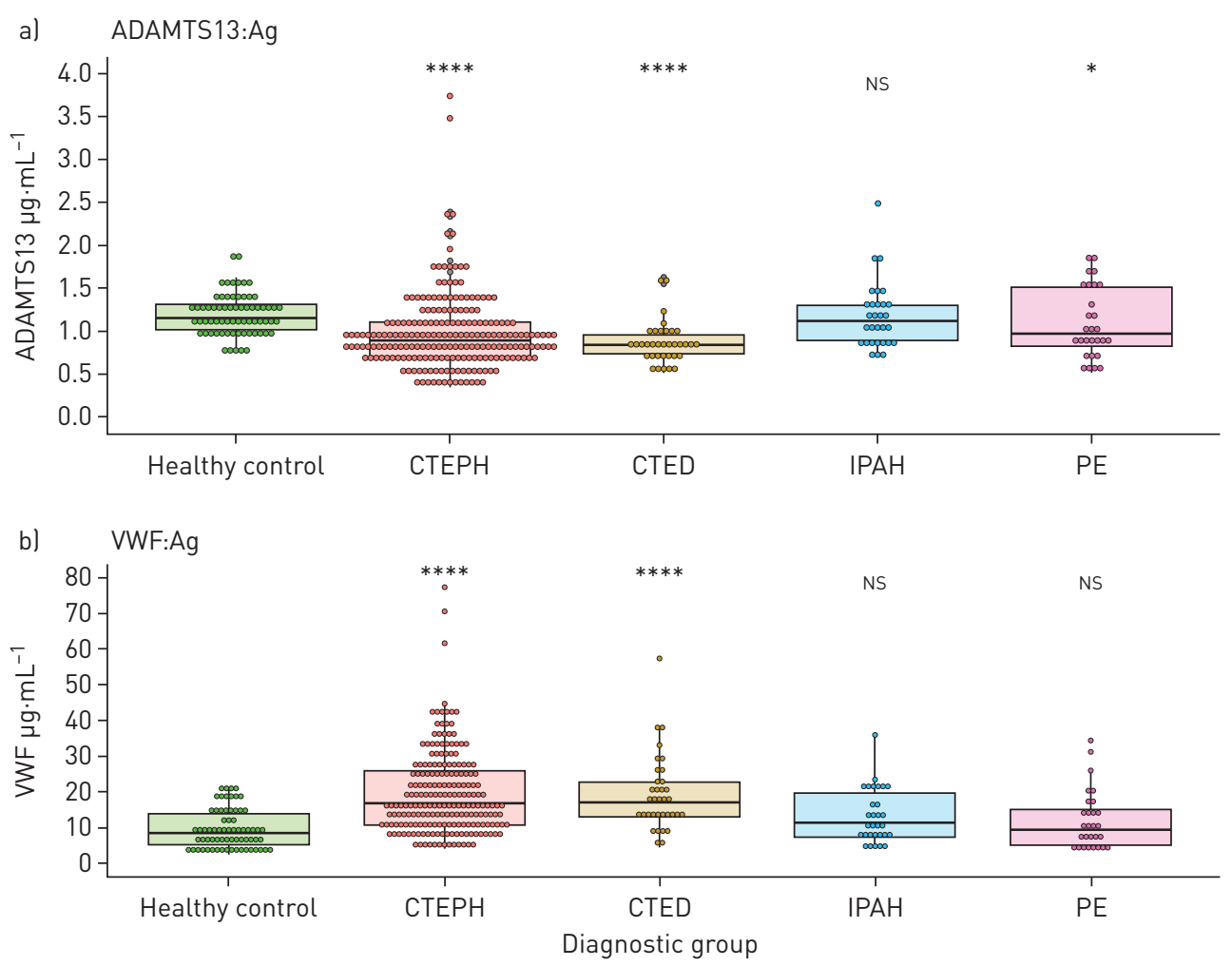

FIGURE 1 a) ADAMTS13 and b) von Willebrand factor (VWF) antigen (Ag) levels by diagnostic group. Dunn's test with false discovery rate adjustment was used to calculate $p$-values. Healthy control, $n=68$; chronic thromboembolic pulmonary hypertension (CTEPH), $n=208$; chronic thromboembolic disease (CTED), $n=35$; idiopathic pulmonary arterial hypertension (IPAH), $n=28$; pulmonary embolism (PE), $n=28 .{ }^{*}: p \leqslant 0.05$; ${ }^{* *}: p \leqslant 0.01 ;{ }^{* * *}: p \leqslant 0.001 ;{ }^{* * * *}: p \leqslant 0.0001 ;$ NS: nonsignificant.

\section{VWF plasma concentrations}

We confirmed that VWF antigen levels are increased in CTEPH $\left(16.7(15.2) \mu \mathrm{g} \cdot \mathrm{mL}^{-1}, \mathrm{p}<0.001\right)$ compared to healthy controls $\left(8.45(8.77) \mu \mathrm{g} \cdot \mathrm{mL}^{-1}\right.$ ) (figure $1 \mathrm{~b}$ ). Furthermore, VWF levels were increased in CTED (17.0 (10.1) $\left.\mu \mathrm{g} \cdot \mathrm{mL}^{-1}, \mathrm{p}<0.001\right)$ compared to healthy controls, but were no different to those in CTEPH $(\mathrm{p}=0.834)$ (supplementary table S2). There was no difference in VWF antigen levels between healthy controls and patients with IPAH (11.6 (12.3) $\left.\mu \mathrm{g} \cdot \mathrm{mL}^{-1}, \mathrm{p}=0.071\right)$ or PE $\left(9.23(9.82) \mu \mathrm{g} \cdot \mathrm{mL}^{-1}, \mathrm{p}=0.433\right)$.

Multivariable linear regression was also used for VWF plasma concentrations as described for ADAMTS13. This confirmed that VWF was significantly increased in the CTEPH $(\beta+75.5 \%, 95 \%$ CI $44.8-113 \%, \mathrm{p}<0.001)$ and CTED $(\beta+89.5 \%, 95 \%$ CI $48.0-143 \%, \mathrm{p}<0.001)$ groups (supplementary table S4). VWF plasma concentrations were not significantly associated with IPAH, PE, sex or ethnicity.

The combination of low ADAMTS13 and high VWF antigen levels had a synergistic effect on the odds of CTEPH (OR 14.5, 95\% CI 5.33-47.4, p<0.001) compared with healthy controls (supplementary figure S2 and table S5).

ADAMTS13 and VWF: pre- and post-pulmonary endarterectomy

Matched samples were taken post-PEA from 22 CTEPH patients after a median of 343 (216) days. There were no differences in levels of ADAMTS13 (median of differences (IQR) $-0.0328(0.250) \mu \mathrm{g} \cdot \mathrm{mL}^{-1}$, $\mathrm{p}=0.777)$ or VWF protein $\left(-3.05(10.7) \mu \mathrm{g} \cdot \mathrm{mL}^{-1}, \mathrm{p}=0.777\right)$ following removal of proximal organised thrombus material by PEA (figure 2).

\section{ADAMTS13 activity, D-dimer, anti-ADAMTS13 autoantibodies and VWF multimers}

Specific ADAMTS13 activity (activity:antigen (Act:Ag) ratio) was increased in CTEPH (Act:Ag 1.57 (0.32)) compared with healthy controls (1.05 (0.190), $\mathrm{p}<0.001)$ (figure $3 \mathrm{a})$.

Plasmin and thrombin are able to inactivate ADAMTS13 proteolytically in vitro and plasmin-mediated ADAMTS13 cleavage has been observed in TTP [27, 28]. Furthermore, abnormalities in the fibrinolysis pathway have been implicated in CTEPH [4]. Therefore, we used fibrinogen degradation products 

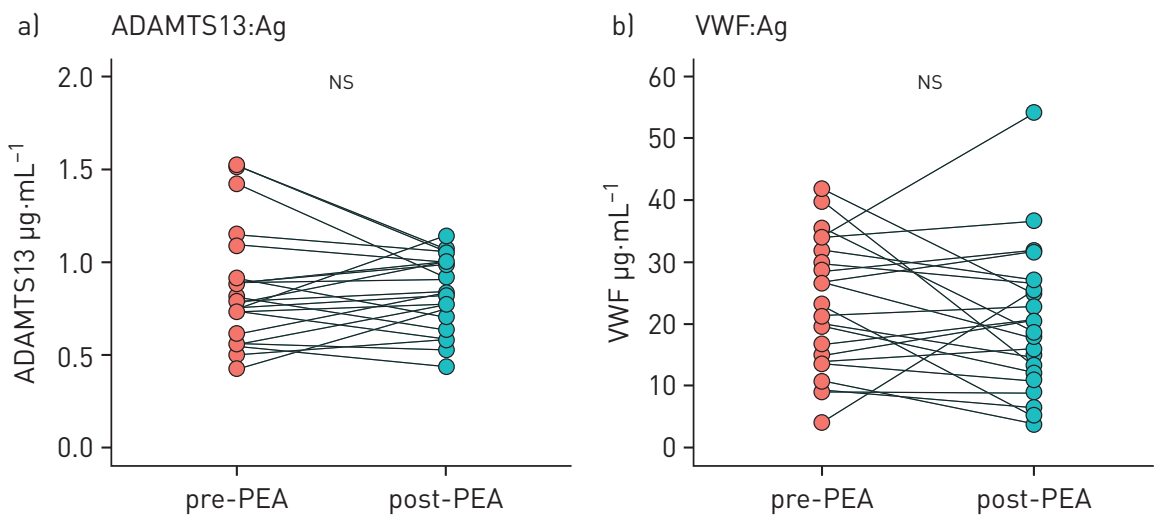

FIGURE 2 a) ADAMTS13 and b) von Willebrand factor (VWF) antigen (Ag) levels pre- and post-pulmonary endarterectomy (PEA) ( $n=22)$. Wilcoxon signed-rank test was used to calculate $p$-values. Ns: nonsignificant.

measured by $\mathrm{D}$-dimer as a potential surrogate marker of plasmin and thrombin activity. D-dimer was increased in CTEPH $\left(1.24(1.25) \mu \mathrm{g} \cdot \mathrm{mL}^{-1}\right)$ compared to healthy controls $\left(0.538(0.344) \mu \mathrm{g} \cdot \mathrm{mL}^{-1}, \mathrm{p}=0.030\right)$ (figure 3b). Specific ADAMTS13 activity was not correlated with D-dimer in the CTEPH (rho=0.0938, $\mathrm{p}=0.761)$ or healthy control ( $\mathrm{rho}=-0.220, \mathrm{p}=0.313$ ) groups (figure $3 \mathrm{c}$ ).

Because the ADAMT13 reduction in TTP has an autoimmune mechanism, we investigated whether anti-ADAMTS13 autoantibodies are increased in CTEPH. There was no significant difference in
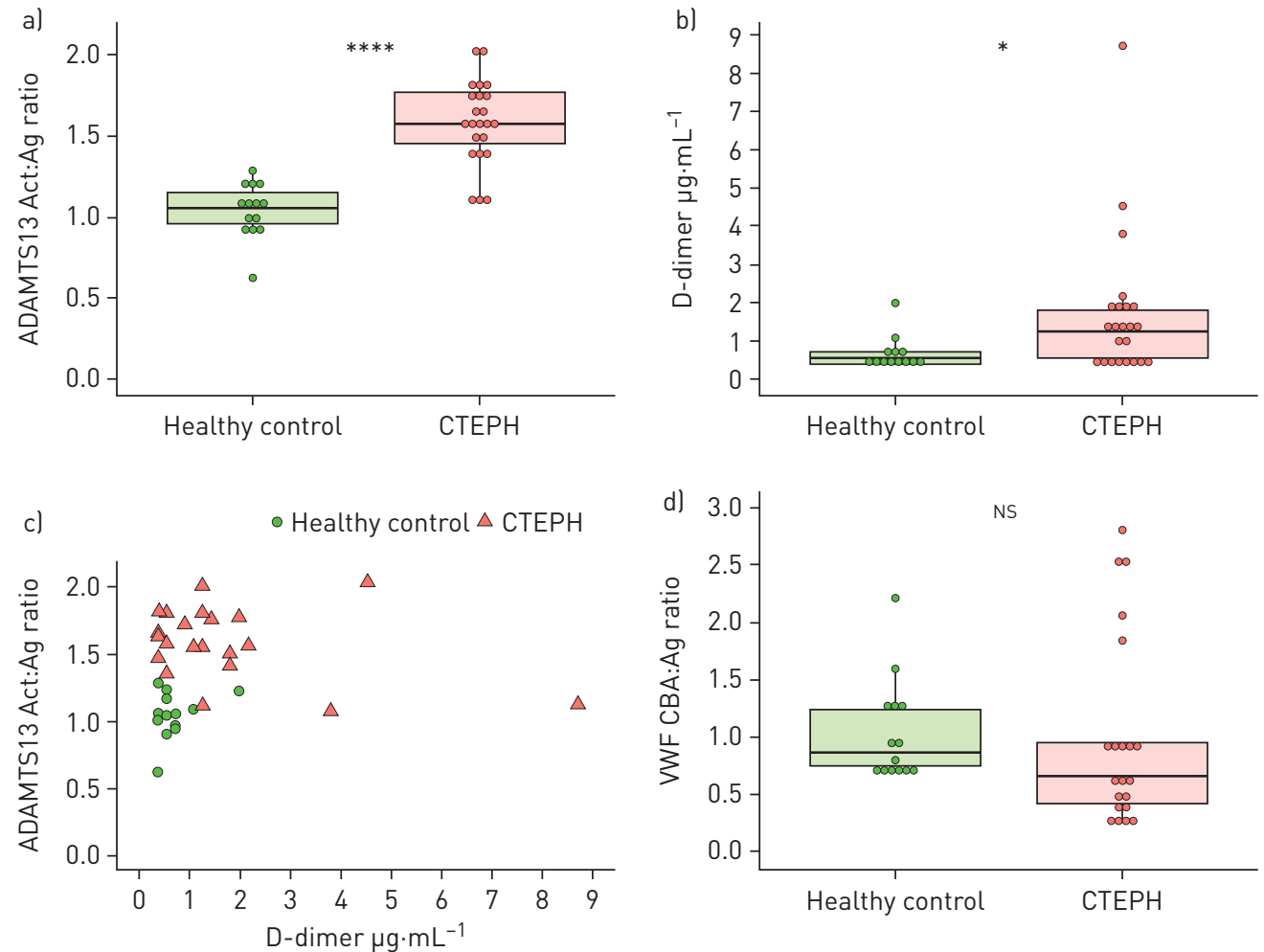

FIGURE 3 ADAMTS13 activity, D-dimer and von Willebrand factor (VWF) multimeric size in chronic thromboembolic pulmonary hypertension (CTEPH) and healthy controls. a) Specific ADAMTS13 activity (activity:antigen (Act:Ag) ratio). b) D-dimer antigen levels. c) Specific ADAMTS13 activity and D-dimer antigen correlation. Healthy control correlation: rho $=0.0938, p=0.761 ;$ CTEPH correlation: rho $=-0.220, p=0.313$. d) VWF multimeric size (VWF collagen binding assay (CBA):Ag ratio). A subset of CTEPH patients ( $n=23$ ) with the lowest ADAMTS13 antigen levels (below the first quartile of the CTEPH group) and healthy controls $(n=14$ ) were used for $a-c$. VWF multimeric size was measured in CTEPH ( $n=21)$ samples with the highest VWF antigen concentrations (above the third quartile of the CTEPH group) using the same healthy control subset and displayed in $d$. The Mann-Whitney $U$ test was used to calculate group differences ( $a, b, d)$ and correlation was assessed using Spearman's rank correlation coefficients (c). 
anti-ADAMTS13 autoantibodies between CTEPH (92.3\% (38.9\%)) and healthy controls (76.0\% (16.5\%), $\mathrm{p}=0.180$ ) (supplementary figure $\mathrm{S} 3$ ).

We hypothesised that a decrease in ADAMTS13 antigen levels would result in reduced VWF cleavage and an increase in high multimeric VWF, as occurs in TTP [29]. There was no difference in VWF multimeric size between CTEPH (VWF CBA:Ag ratio, 0.659 (0.537)) and healthy controls (0.866 (0.494), p=0.160) (figure $3 \mathrm{~d}$ ).

\section{Clinical phenotype associations with ADAMTS13 and VWF}

In CTEPH, ADAMTS13 and VWF did not significantly correlate with markers of disease severity (6MWD, pulmonary vascular resistance or $\mathrm{N}$-terminal pro-brain natriuretic peptide) (supplementary figure S4). Given that inflammation has been associated with both CTEPH and abnormalities in the ADAMTS13VWF axis, we investigated if they were correlated [30, 31]. There were no correlations with blood markers of inflammation (C-reactive protein, white cell count, neutrophil and lymphocyte percentages) (supplementary figure S5).

\section{ABO groups and ADAMTS13-VWF}

There was no difference in ADAMTS13 antigen levels when stratified by simple genetic $A B O$ groups $(\mathrm{O}, \mathrm{A}, \mathrm{B}, \mathrm{AB} ; \mathrm{p}=0.443$ ) (figure $4 \mathrm{a}$ ) or more comprehensive genetic $A B O$ groups $(\mathrm{p}=0.616$ ) (supplementary figure S6a).

VWF levels did not vary by $A B O$ group (figure $4 \mathrm{~b}$ and supplementary figure S6b); however, when accounting for covariates (supplementary table S6), $A B O$ group B had a higher VWF level $(\beta+51.3 \%, 95 \%$ CI $5.30-117 \%, \mathrm{p}<0.001)$ than group O. $A B O$ group A also had a higher VWF level, although this was not statistically significant $(\beta+19.8 \%, 95 \%$ CI $-1.75-46.1 \%, \mathrm{p}=0.073)$. Patients with $A B O$ group $\mathrm{O}$ had the lowest VWF levels within the CTEPH group $\left(14.5(13.0) \mu \mathrm{g} \cdot \mathrm{mL}^{-1}\right)$, which were still significantly higher than those of healthy controls $\left(8.45(8.77) \mu \mathrm{g} \cdot \mathrm{mL}^{-1}, \mathrm{p}<0.001\right)$.

a)

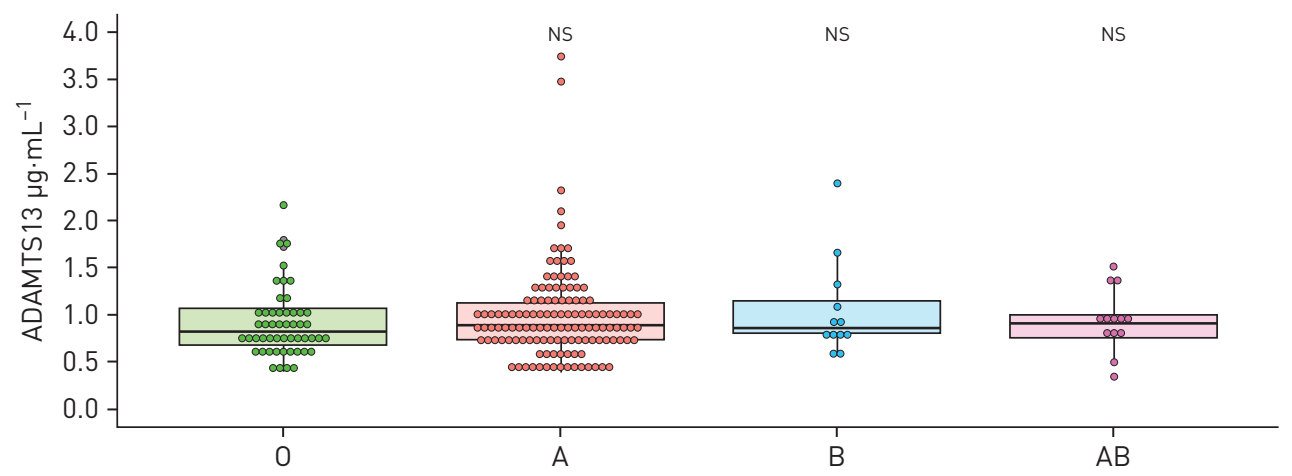

b)

VWF:Ag

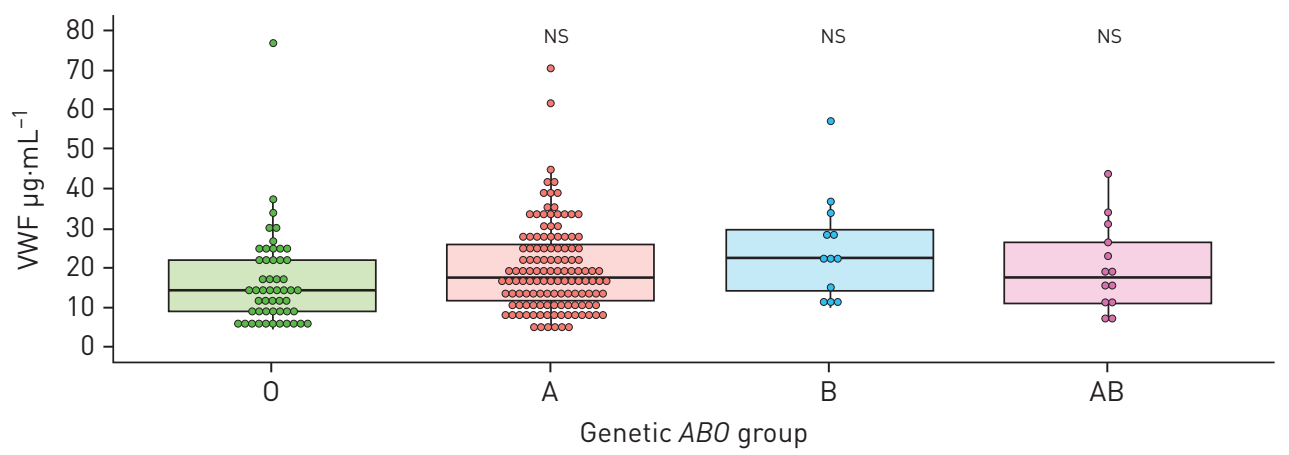

FIGURE 4 ADAMTS13 and von Willebrand factor (VWF) antigen (Ag) levels by $A B O$ genetic groups. Chronic thromboembolic pulmonary hypertension (CTEPH) $(n=182)$ and chronic thromboembolic disease (CTED) ( $n=22$ ) patients with genotypes and protein levels available (in $n=3$, a genetic $A B O$ group could not be inferred) were included. Dunn's test with false discovery rate adjustment was used to calculate $p$-values. 0 group, $n=51 ; A$ group, $n=128 ; B$ group, $n=12 ; A B$ group, $n=13$. 
There was no difference in ADAMTS13 antigen levels between $A B O$ groups when accounting for covariates with multivariable linear regression.

\section{Protein quantitative trait loci for ADAMTS13}

There were five SNPs in the ADAMTS13 $\pm 40 \mathrm{~kb}$ region that were significantly associated with ADAMTS13 protein in a multivariable linear regression model (supplementary table S7). The most significant SNP (rs3739893, risk allele C, $\beta-37.1 \%, 95 \% \mathrm{CI}-48.1--23.8 \%, \mathrm{p}=3.78 \times 10^{-06}$ ) is a $5^{\prime}$ untranslated region variant in the C9orf96 gene, which is $\sim 8 \mathrm{~kb} 5^{\prime}$ of the ADAMTS13 gene. In a model adjusted for age, sex and batch, the lead SNP (rs3739893) explained 7.7\% of the variance in ADAMTS13 levels within the CTEPH group (supplementary table S8). In the whole CTEPH GWAS, the effect allele frequency for rs3739893 in CTEPH cases (0.0128) and healthy controls (0.0158) was not significantly different, which suggests that it is not associated with CTEPH disease risk.

\section{Discussion}

This is the first study demonstrating a marked reduction in plasma levels of ADAMTS13 in CTEPH. This is independent of pulmonary hypertension, disease severity or systemic inflammation. We confirm that VWF is increased in CTEPH and implicate dysregulation of the ADAMTS13-VWF axis in CTEPH pathobiology.

The magnitude of ADAMTS13 reduction and VWF increase in CTEPH is greater than observed in studies of ischaemic stroke using the same methodology [23]. Furthermore, levels are lower in CTEPH than in CAD when considering the proportion of patients in the lowest ADAMTS13 quartile (65\% versus 28\%, respectively) [13]. Additionally, the combination of decreased ADAMTS13 and increased VWF has a synergistic effect on the odds of CTEPH that is greater than observed in CAD or ischaemic stroke [23]. The more pronounced ADAMTS13-VWF dysregulation in CTEPH may reflect the larger surface area of the vascular endothelium involved or, alternatively, that ADAMTS13-VWF dysregulation is more important in CTEPH pathobiology. Although ADAMTS13 is predominately produced by the liver, the contribution to plasma levels from vascular endothelial cells could be substantial given the large surface area of the lung vasculature [9]. A reciprocal relationship has previously been described between ADAMTS13 and VWF $[32,33]$. The reduction in ADAMTS13 remained in our study when VWF levels were adjusted for, which is consistent with low ADAMTS13 being an independent risk factor in other thrombotic diseases [11].

Following PEA and removal of proximal thromboembolic material, the ADAMTS13-VWF axis remained dysregulated despite normalisation of haemodynamic parameters. Additionally, there was equal perturbation of the axis in CTED, and no correlation with CTEPH disease severity, confirming that the changes are not due to the presence of pulmonary hypertension or organised thrombus per se. Interestingly, there was no abnormality in ADAMTS13 levels in IPAH despite this group having higher pulmonary vascular resistance, implying that distal pulmonary artery endothelial dysfunction and small vessel vasculopathy are not responsible [34]. Taken together, these observations demonstrate dysregulation of the ADAMTS13-VWF axis in CTEPH pathogenesis.

Low ADAMTS13 levels could be driven by the activation of fibrinolytic pathways and an increase in thrombin and/or plasmin, which have the potential to proteolytically inactivate ADAMTS13 [27]. D-dimer was raised in CTEPH, though there was no correlation with ADAMTS13. High multimeric forms of VWF do not appear to be increased in CTEPH. This is surprising, because increased high multimeric VWF occurs when ADAMTS13 is reduced in TTP and has been suggested to occur in ischaemic stroke and CAD [23, 29]. Measuring VWF multimeric size systemically may not reflect the local disease microenvironment in the pulmonary vascular endothelium. Additionally, the localised flow conditions that may be altered in CTEPH are important in VWF structure, cleavage by ADAMTS13 and thrombus resolution [35]. The increase in specific ADAMTS13 activity in CTEPH may reflect an increased conformational activation of ADAMTS13 by its substrate VWF, due to the altered ADAMTS13:VWF ratio [36].

The $A B O$ gene is located in close proximity to and modest linkage disequilibrium with the ADAMTS13 gene, raising the possibility that $A B O$ may influence the ADAMTS13-VWF axis. ABO blood groups are associated with CTEPH, with an over-representation of the non-O groups [20]. Genetic variation in $A B O$ has also been associated with ischaemic stroke, CAD and venous thromboembolism [37, 38]. The proposed mechanism of this association has been via VWF plasma levels, which are $25 \%$ higher in non-O individuals [39]. We found that VWF was increased in some non-O groups within CTEPH; however, VWF was still significantly higher in the CTEPH O group than in healthy controls. This implies that there are additional contributing causes for the increased VWF in CTEPH. Conversely, $A B O$ is a pleiotropic 
locus and may have alternative functional effects in CTEPH, including mediating pathways involved in inflammation and angiogenesis [26].

We identified a pQTL (rs3739893) in the C9orf96 gene ( $\sim \mathrm{kb} 5^{\prime}$ of the ADAMTS13 gene) that is associated with ADAMTS13 protein levels and has been described in two previous studies [18, 25]. In a GWAS of ADAMTS13 antigen levels in a healthy cohort, this SNP was significantly associated with a similar effect size $(\beta-22.3 \%)$. Whilst this confirms that ADAMTS13 protein is genetically regulated, this SNP only accounts for a modest variance of $\sim 8 \%$ in ADAMTS13 protein levels and is not primarily associated with CTEPH disease risk.

A strength of this study is that we investigated the ADAMTS13-VWF axis in a spectrum of thromboembolic disease from acute PE to chronic thromboembolism with and without pulmonary hypertension. Our study contains a large sample of well-characterised CTEPH patients who have been extensively phenotyped in an experienced national CTEPH centre. ADAMTS13-VWF imbalance does not occur in PE when assessed by multivariable regression, although we were underpowered to detect smaller effect sizes. This raises an intriguing possibility, that there are differences in the ADAMTS13-VWF axis in the spectrum of thromboembolic disease. Future studies using robustly phenotyped PE cohorts to ascertain the presence and extent of residual perfusion defects should investigate if the ADAMTS13-VWF axis varies in post-PE syndrome. Clinical prediction scores for CTEPH following acute PE do not currently incorporate biomarkers [40]. Determining if dysregulation of the ADAMTS13-VWF axis precedes the development of chronic thromboembolic pathology could inform CTEPH risk stratification.

In summary, we report that the ADAMTS13-VWF axis is dysregulated in CTEPH and this is unrelated to pulmonary hypertension, disease severity or systemic inflammation. This implicates the ADAMTS13VWF axis in CTEPH pathogenesis.

Acknowledgements: We would like to acknowledge the help of participating pulmonary hypertension centres (Royal Papworth Hospital, Hammersmith Hospital and the GWAS consortium), research nurses and clinical staff involved in the recruitment of patients. We thank the patients and their families who were recruited to this study. This work was supported by the NIHR Cambridge BRC and an unrestricted research grant from Bayer Pharmaceuticals. MN was supported by funding from a British Heart Foundation Scholarship.

Conflict of interest: M. Newnham reports education support (travel, registration and accommodation) to attend conferences from MSD and GSK, outside the submitted work. K. South has nothing to disclose. M. Bleda has nothing to disclose. W.R. Auger reports grants and non-financial support for advisory board work (travel support only) from Bayer, outside the submitted work. J.A. Barberà has nothing to disclose. H. Bogaard has nothing to disclose. K. Bunclark has nothing to disclose. J.E. Cannon reports grants for educational meetings from Actelion, GSK and MSD, outside the submitted work. M. Delcroix has nothing to disclose. C. Hadinnapola has nothing to disclose. L.S. Howard reports grants from Bayer PLC, during the conduct of the study. D. Jenkins reports grants and personal fees from Bayer, and personal fees from Actelion, outside the submitted work. E. Mayer reports speaker and consultancy fees from Actelion, Bayer and MSD, and speaker fees from Pfizer, outside the submitted work. C. Ng has nothing to disclose. C.J. Rhodes has nothing to disclose. N. Screaton has nothing to disclose. K. Sheares reports educational support (travel, registration and accommodation) to attend conferences from Actelion, Bayer, MSD and GSK, and has been on an advisory board for Actelion, outside the submitted work. M.A. Simpson has a contract of service with Genomics PLC, outside the submitted work. M. Southwood has nothing to disclose. L. Su has nothing to disclose. D. Taboada reports speaker honoraria and education/travel grants from Actelion, Bayer, GlaxoSmithKline, Lilly, MDS and Pfizer, outside the submitted work. M. Traylor has nothing to disclose. R.C. Trembath has nothing to disclose. S.S. Villar has nothing to disclose. M.R. Wilkins has nothing to disclose. J. Wharton reports personal fees for advisory board work from Actelion Pharmaceuticals Ltd, outside the submitted work. S. Gräf has nothing to disclose. J. Pepke-Zaba (or her institution) has received research/educational grants and has been serving on advisory boards for Actelion, Bayer, Merck and GSK. M. Laffan reports grants from British Heart Foundation during the conduct of the study; and support to attend conferences from Shire, outside the submitted work. D.A. Lane has nothing to disclose. N.W. Morrell has nothing to disclose. M. Toshner reports grants and personal fees from Bayer, Merck and Actelion, personal fees from GSK, and grants from Roche, during the conduct of the study.

\section{References}

1 Ende-Verhaar YM, Cannegieter SC, Vonk Noordegraaf A, et al. Incidence of chronic thromboembolic pulmonary hypertension after acute pulmonary embolism: a contemporary view of the published literature. Eur Respir J 2017; 49: 1601792.

2 Moser KM, Bloor CM. Pulmonary vascular lesions occurring in patients with chronic major vessel thromboembolic pulmonary hypertension. Chest 1993; 103: 685-692.

3 Galiè N, Kim HS. Pulmonary microvascular disease in chronic thromboembolic pulmonary hypertension. Proc Am Thorac Soc 2006; 3: 571.

4 Morris TA, Marsh JJ, Chiles PG, et al. Fibrin derived from patients with chronic thromboembolic pulmonary hypertension is resistant to lysis. Am J Respir Crit Care Med 2006; 173: 1270-1275.

5 Satoh T, Satoh K, Yaoita N, et al. Activated TAFI promotes the development of chronic thromboembolic pulmonary hypertension: a possible novel therapeutic target. Circ Res 2017; 120: 1246-1262.

6 Bonderman D, Turecek PL, Jakowitsch J, et al. High prevalence of elevated clotting factor VIII in chronic thromboembolic pulmonary hypertension. Thromb Haemost 2003; 90: 372-376.

7 Sadler JE. Biochemistry and genetics of von Willebrand factor. Annu Rev Biochem 1998; 67: 395-424. 
Dong JF, Moake JL, Nolasco L, et al. ADAMTS-13 rapidly cleaves newly secreted ultralarge von Willebrand factor multimers on the endothelial surface under flowing conditions. Blood 2002; 100: 4033-4039.

9 Zheng XL. Structure-function and regulation of ADAMTS-13 protease. J Thromb Haemost 2013; 11: Suppl. 1, $11-23$.

10 Levy GG, Nichols WC, Lian EC, et al. Mutations in a member of the ADAMTS gene family cause thrombotic thrombocytopenic purpura. Nature 2001; 413: 488-494.

11 Sonneveld MA, de Maat MP, Leebeek FW. Von Willebrand factor and ADAMTS13 in arterial thrombosis: a systematic review and meta-analysis. Blood Rev 2014; 28: 167-178.

12 Tsai AW, Cushman M, Rosamond WD, et al. Coagulation factors, inflammation markers, and venous thromboembolism: the longitudinal investigation of thromboembolism etiology (LITE). Am J Med 2002; 113: 636-642.

13 Maino A, Siegerink B, Lotta LA, et al. Plasma ADAMTS-13 levels and the risk of myocardial infarction: an individual patient data meta-analysis. I Thromb Haemost 2015; 13: 1396-1404.

14 Mazetto BM, Orsi FL, Barnabe A, et al. Increased ADAMTS13 activity in patients with venous thromboembolism. Thromb Res 2012; 130: 889-893.

15 Llobet D, Tirado I, Vilalta N, et al. Low ADAMTS13 levels are associated with venous thrombosis risk in women. Thromb Res 2017; 157: 38-40.

16 Gouvea CP, Matsuda SS, Vaez R, et al. The role of high von Willebrand factor and low ADAMTS13 levels in the risk of venous thromboembolism. Blood 2013; 122: 1128.

17 Orstavik KH, Magnus P, Reisner H, et al. Factor VIII and factor IX in a twin population. Evidence for a major effect of ABO locus on factor VIII level. Am J Hum Genet 1985; 37: 89-101.

18 Ma Q, Jacobi PM, Emmer BT, et al. Genetic variants in ADAMTS13 as well as smoking are major determinants of plasma ADAMTS13 levels. Blood Adv 2017; 1: 1037-1046.

19 Chion CK, Doggen CJ, Crawley JT, et al. ADAMTS13 and von Willebrand factor and the risk of myocardial infarction in men. Blood 2007; 109: 1998-2000.

20 Delcroix M, Lang I, Pepke-Zaba J, et al. Long-term outcome of patients with chronic thromboembolic pulmonary hypertension: results from an international prospective registry. Circulation 2016; 133: 859-871.

21 Galie N, Humbert M, Vachiery JL, et al. 2015 ESC/ERS guidelines for the diagnosis and treatment of pulmonary hypertension: The Joint Task Force for the Diagnosis and Treatment of Pulmonary Hypertension of the European Society of Cardiology (ESC) and the European Respiratory Society (ERS): endorsed by: Association for European Paediatric and Congenital Cardiology (AEPC), International Society for Heart and Lung Transplantation (ISHLT). Eur Heart J 2016; 37: 67-119.

22 Konstantinides SV, Torbicki A, Agnelli G, et al. 2014 ESC guidelines on the diagnosis and management of acute pulmonary embolism. Eur Heart J 2014; 35: 3033-3069.

23 Andersson HM, Siegerink B, Luken BM, et al. High VWF, low ADAMTS13, and oral contraceptives increase the risk of ischemic stroke and myocardial infarction in young women. Blood 2012; 119: 1555-1560.

24 McCarthy S, Das S, Kretzschmar W, et al. A reference panel of 64,976 haplotypes for genotype imputation. Nat Genet 2016; 48: 1279-1283.

25 de Vries PS, Boender J, Sonneveld MA, et al. Genetic variants in the ADAMTS13 and SUPT3H genes are associated with ADAMTS13 activity. Blood 2015; 125: 3949-3955.

26 Suhre K, Arnold M, Bhagwat AM, et al. Connecting genetic risk to disease end points through the human blood plasma proteome. Nat Commun 2017; 8: 14357

27 Crawley JT, Lam JK, Rance JB, et al. Proteolytic inactivation of ADAMTS13 by thrombin and plasmin. Blood 2005; 105: 1085-1093.

28 Feys HB, Vandeputte N, Palla R, et al. Inactivation of ADAMTS13 by plasmin as a potential cause of thrombotic thrombocytopenic purpura. J Thromb Haemost 2010; 8: 2053-2062.

29 Moake JL, Rudy CK, Troll JH, et al. Unusually large plasma factor VIII:von Willebrand factor multimers in chronic relapsing thrombotic thrombocytopenic purpura. N Engl J Med 1982; 307: 1432-1435.

30 Quarck R, Wynants M, Verbeken E, et al. Contribution of inflammation and impaired angiogenesis to the pathobiology of chronic thromboembolic pulmonary hypertension. Eur Respir J 2015; 46: 431-443.

31 Schwameis M, Schorgenhofer C, Assinger A, et al. VWF excess and ADAMTS13 deficiency: a unifying pathomechanism linking inflammation to thrombosis in DIC, malaria, and TTP. Thromb Haemost 2015; 113: 708-718.

32 Mannucci PM, Capoferri C, Canciani MT. Plasma levels of von Willebrand factor regulate ADAMTS-13, its major cleaving protease. Br J Haematol 2004; 126: 213-218.

33 Reiter RA, Varadi K, Turecek PL, et al. Changes in ADAMTS13 (von-Willebrand-factor-cleaving protease) activity after induced release of von Willebrand factor during acute systemic inflammation. Thromb Haemost 2005; 93 : 554-558.

34 Wolff B, Lodziewski S, Bollmann T, et al. Impaired peripheral endothelial function in severe idiopathic pulmonary hypertension correlates with the pulmonary vascular response to inhaled iloprost. Am Heart J 2007; 153: 1081-1087.

35 Baldauf C, Schneppenheim R, Stacklies W, et al. Shear-induced unfolding activates von Willebrand factor A2 domain for proteolysis. J Thromb Haemost 2009; 7: 2096-2105.

36 South K, Freitas MO, Lane DA. A model for the conformational activation of the structurally quiescent metalloprotease ADAMTS13 by von Willebrand factor. J Biol Chem 2017; 292: 5760-5769.

37 Dichgans M, Malik R, Konig IR, et al. Shared genetic susceptibility to ischemic stroke and coronary artery disease: a genome-wide analysis of common variants. Stroke 2014; 45: 24-36.

38 Germain M, Chasman DI, de Haan H, et al. Meta-analysis of 65,734 individuals identifies TSPAN15 and SLC44A2 as two susceptibility loci for venous thromboembolism. Am J Hum Genet 2015; 96: 532-542.

39 Gill JC, Endres-Brooks J, Bauer PJ, et al. The effect of ABO blood group on the diagnosis of von Willebrand disease. Blood 1987; 69: 1691-1695.

40 Klok FA, Dzikowska-Diduch O, Kostrubiec M, et al. Derivation of a clinical prediction score for chronic thromboembolic pulmonary hypertension after acute pulmonary embolism. J Thromb Haemost 2016; 14: 121-128. 